\title{
A Comparative Performance Analysis of PID Control and Sliding Mode Control of Two Link Robot Manipulator
}

Priyanka Singh ${ }^{1}$, L.B Prasad ${ }^{2}$

${ }^{1}$ Priyanka Singh, Department of Electrical Engineering, Madan Mohan Malaviya University of Technology, Gorakhpur, U.P, India

${ }^{2}$ L.B Prasad, Department of Electrical Engineering, Madan Mohan Malaviya University of Technology, Gorakhpur, U.P, India

Iprynksngh643@gmail.com, ${ }^{2}$ lpbee@mmmut.ac.in

\section{Abstract}

The paper deals with study of SMC for a robot manipulator. The property of the robot manipulator has low sensitivity to distortions and more efficient than other controllers. The tracking control difficulty for two-link robot manipulator which obeys the Lyapunov stability theorem, the back stepping control (BC) and (SMC) is denoted. The comparison of PID controller and SMC controller has been done by the application of Two-link robot manipulator by using MATLAB technique by constructing simulink models. The stability has been elaborated by comparing the performance of the PID and SMC and investigating the most efficient controller.

Keywords: Lyapunov stability theorem, PID, SMC, Link robot manipulator, Variable Structure System

\section{Introduction}

Today, the main focus in electrical engineering research is on control theory from the 1970s and 1980s, as can be seen from the paper. The robust regulator is the most stable and resistant to deformation between the device and the model. One of the most robust methodologies evolving in this era, called variable structure control, is the VSC approach [9-10]. National observers and tracking system, flexible schemes, model reference system to help design robust controllers. The concept has been efficiently applicable to difficulties such as electric motors, chemical processes, observers, mechanical systems, and signal reconstruction $[2,4$, and 11$]$.

The $S M C$ system becomes an operational mode in control systems with the help of sensitivity and reduction property we can change parameters of plants. Almost every design methodologies for VSS are on the basis of $S M C$. Because of its reduced properties, low sensitivity to distortion and changes in plant parameters, SMC is most effective controller to control the complex type of high-class dynamic plants that operate in unstable situations, which are generally used for many modern technology processes. : era. The main disadvantage of SMC is the conversational approach. This type of approach involves a variation of the control signal, which is associated with a certain frequency, the uninterrupted tendency of the control approach to amplitude, which is able to control or distort the system.

High frequency fluctuations of the control signal, which are directly related to stability of the SMC. However, in real-time applications, the frequency 
fluctuations of control signals and amplitude are obvious, so that the follower falls. The two main conclusions were discussed. Then there is the fast dynamics in the fast-paced management circuit, which can be overlooked in the system model, which is excited by the SMC review. Second, the attractiveness of manufacturers is listed numerically in the microcontroller in case of stable test loss, which can be done on different connections. Use a variety of options or programs to minimize the impact of the conversation. [8,11] Work with complex mechanical systems that have non-linear dynamics. Then the nonlinear control of the control is necessary so that the high selection operation has the maximum power.

Modeling of 2-DOF Robot Arm and Control [2].PID Control applied to a robotic manipulator arm with two DOF and design of dynamic model.[3] A two link robotic manipulator which is a classic example studied in introductory robotic courses. The equations of the two arm are describe by nonlinear differential equation. Because closed loop form are not present, the equation of motion are numerically studied using a numerical method.[4] A conventional SMC and chattering free improved $S M C$ design approach for nonlinear systems. Chattering in conventional $S M C$ can be easily removed by using continuous function in place of discontinuous sign function. As it is not model based controller, the difficulty related to mathematical modeling process is less. [23] Robot manipulator is a link of many links that couple with help of joints. Robotic system and mathematical model to explain or define the behavior of system; such as dynamic linear or non linear to construct the designs of system. Goal of $S M C$ is provide system a angular motion with use of link. [22]

This paper is used in the following way. Section II develops a dynamic model of a robot manipulator. Section III proposed control schemes. Section IV discussed the simulation part. Sections $V$ of the conclusion and future sides have been developed, followed by acknowledgement.

\section{Mathematical Modeling of Two Link Robot Manipulator}

There is a huge amount of difficulty in managing mechanical systems, which is the basis for controlling the current area or the location of the mass using force, otherwise the moment, because of the input variability. The approach can be explained by the following example, which is a robotic arm or can be called a robotic manipulator with $\mathrm{n}$ relations, which is related to the forces with $\mathrm{n}$ joints. The large variety of control questions for square measurement of mechanical systems ensures the position of the dominant position or the location of the force or mass involved, as the effort is variable. The standard exemplar may be a automation arm before robot manipulator with $\mathrm{n}$ links associated by $n$ joints amid input forces/torques that square measure output of actuator, typically electrical actuator, with their personal advanced dynamic.

The actuator dynamics can be left out on top of things designed for electromechanical scheme, suppose they are positive compared to the inertial dynamics of masses. In addition, the different dynamics of structural flexibility are regularly lacking when developing a basic model for a mechanical device. In fact, the difficulty of conversation .The dynamic model can be pictured as a space for a sequential mass of the subtlety of a system of robotic robots $[1,5]$.

$$
M(q) \ddot{q}+N(q, \dot{q}) \dot{q}+F_{v}(\dot{q})+G(q)=[1]
$$

$q \in R^{n x 1}$ Specifies the joint mechanization variables (translational or rational) of the $n$ robot links; $M(q,) \in R^{n \times n}$ is the symmetric positive definite inertia mass matrix; $N(q, \dot{q}) \in R^{n \times n}$ includes Coriolis and centripetal forces; vector, $F_{v}(\dot{q}) \in R^{n \times n}$ elaborates viscous friction ; $G(q) \in R^{n \times n}$ vector includes gravity terms; $\tau \in R^{n \times n}$ is control torque vector.

Define a $2 n$-dimensional state vector $x$ as:

$$
x=\left[\begin{array}{l}
x_{1} \\
x_{2}
\end{array}\right]=\left[\begin{array}{l}
q \\
\dot{q}
\end{array}\right][2]
$$

Then nonlinear plant in question (1) is described as follow:

$$
\dot{x}=A(x)+B(x) u[3]
$$


Where

$$
\begin{gathered}
u=\tau, \\
A(x)=\left[\begin{array}{c}
x_{2} \\
-M^{-1}\left(x_{1}\right)\left[N(x) x_{2}+F_{v}\left(x_{2}\right)+G\left(x_{1}\right)\right]
\end{array}\right]
\end{gathered}
$$$$
\text { [4] }
$$

$$
B(x)=\left[\begin{array}{c}
0 \\
M^{-1}\left(x_{1}\right)
\end{array}\right][5]
$$

This is 2 nth-order system having $n$ input.

\section{CONTROL SCHEMES}

\subsection{Sliding Mode Control}

VSC systems comprise a collection of different, typically quite easy, feedback manipulate legal guidelines and a choice rule. Relying on the popularity of the device, a selection rule, frequently termed the switching characteristic, determines which of control legal guidelines is "on line" at any individual time. The temporary dynamics of a VSC machine consists of two modes: a "attaining mode" (or "no sliding mode"), follow by using a "sliding mode". Therefore layout of VSC includes, first, design of right $n$ dimensional switching function $s(x)$ for a favored sliding mode dynamics, and 2 nd, design of a manage for achieving mode such that a attaining situation is met. The favored sliding mode dynamics is often a fast and stable error-unfastened response void of overshoot (an asymptotic convergence to final kingdom could be executed in sliding mode). For the attaining mode, desired response normally is to reach switching manifold, defined by way of:

$$
s(x)=c^{T} x=0[6]
$$

In finite time with small overshoot w.r.t switching manifold. For and different input system, there are infinite switching functions and $2 n-1$ sliding manifolds of different dimensions. The first $m$ of them are designated as:

$$
S_{i}=\left\{x \mid s_{i}=c^{T} x=0\right\}, I=1, \ldots, n[7]
$$

Defining the dynamic behavior of the device on the floor is described as the best sliding movement. The benefits of getting one of these motions are twofold. First, there may be a reduction in order, and second, the sliding movement is insensitive to the parameter options included in the input waves. .

Gao and Hung [3] have proposed a method of improvement based on a new system called reaching law technique for the design of nonlinear VSC systems. The technique simultaneously looks at the security of the situation and the security of the situation, the senseless regulation of the general order to make the transition to a free order, the ability of the segment to achieve a dynamic dynamic impact on the car, and approaches the degree of control. The system used in this technique is very simple and smooth to perform, even for non-linear structures. The implementing law is a differential equation that defines the dynamics of the switching function $s(x)$. The asymmetrically strong $s(x)$ differential equation is itself a feasible situation. In addition, the dynamic satisfaction of the VSC system can be controlled by selecting parameters during the differential equation. It is a realistic trendy trend in the implementation of the legislation.

$s=-\dot{Q} \operatorname{sgn}(s)-K h(s)[8]$

Where

$$
\begin{gathered}
Q=\operatorname{diag}\left[q_{1}, \ldots, q_{n}\right], q_{i}>0 \\
\operatorname{sgn}(s)=\left[\operatorname{sgn}\left(s_{1}\right), \ldots, \operatorname{sgn}\left(s_{n}\right)\right]^{T}, \\
K=\operatorname{diag}\left[k_{1}, \ldots, k_{n}\right], k_{i}>0 \\
h(s)=\left[h_{1}\left(s_{1}\right), \ldots, h_{n} s_{n}\right]^{T}, \\
s_{i} h_{i}\left(s_{i}\right)>0, h_{i}(0)=0
\end{gathered}
$$

3 practical special cases of (6) are given below.

1) Constant rate reaching:

$\dot{s}=-Q \operatorname{sgn}(s)[9]$ 
This law requires variable $s(x)$ switches to be switched to variable manifolds $S$ with the following velocity $i q s$. The merit of this mature law is its simplicity, but as can be proved later, if the $q i$ is too small, it will take a long time to reach. However, $q i$ is extremely important for extreme screaming.

2) Constant plus proportional rate reaching:

$\dot{s}=-Q \operatorname{sgn}(s)-k s[10]$

truely, via the usage of which includes the proportional rate time period $K s$, the nation is pressured to method the switching manifolds faster at the same time as $s$ is huge. it is able to established that the accomplishing time for $x$ to move from an initial country $x 0$ to the switching manifold Si is finite, and is given with the aid of:

$T_{i}=\frac{1}{k_{i}} \ln \frac{k_{i}\left|s_{i}\right|+q_{i}}{q_{i}}[11]$

3) Power rate reaching:

$\dot{s}_{l}=-k_{i}\left|s_{i}\right|^{\alpha} \operatorname{sgn}\left(s_{i}\right), 0<\alpha<1, i=1, \ldots, n .[12]$

This reahing law will increase accomplish speed when state is far away from switching manifold, but reduces price while the state is close to manifold. The end result is a fast reaching and low chattering reaching mode. Integrating (10) from

$s_{i}=s_{i 0}$ to $s_{i}=0$ yield:

$T_{i}=\frac{\left|s_{i 0}\right|^{1-\alpha}}{(1-\alpha) k_{i}}, i=1, \ldots, n,[13]$

showing that the reaching time $T_{i}$ is finite.

\subsection{PID Control}

In industrial application PID controllers are highly used control system about $95 \%$ of closed loop system operations of trade automation area used PID controllers. There are three controllers are used joint to perform operation. Feedback controller - it provides manipulated output at the desired input levels.

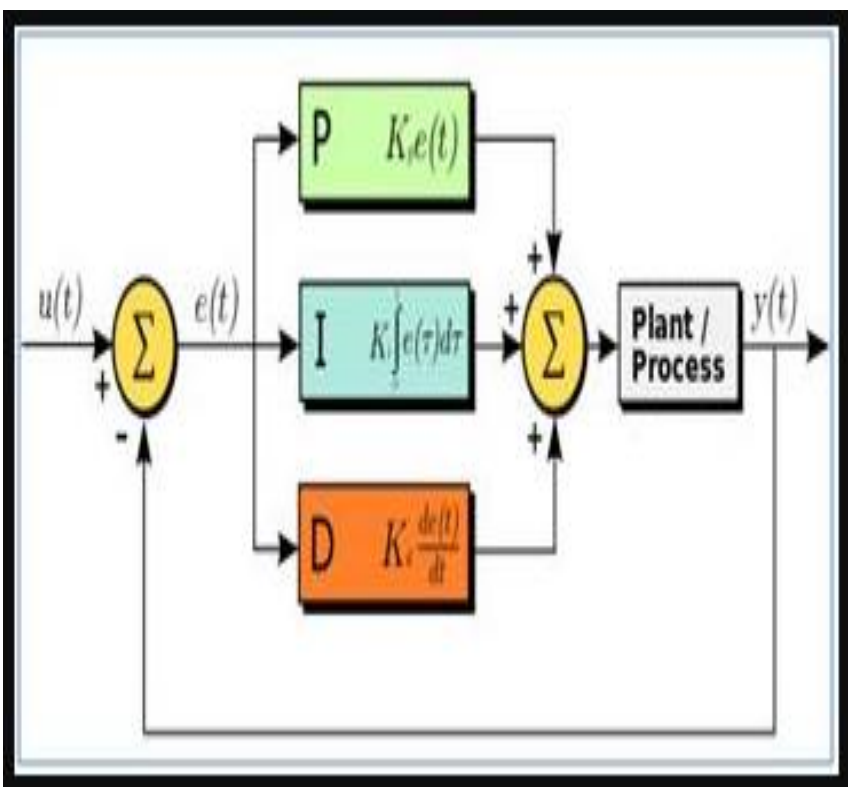

Fig.1 Basic Diagram of PID Controller

As a feedback controller, it supplies the manipulate output at desired tiers. Earlier than microprocessors were invented, PID control was carried out by way of the analog electronic components. However today all PID controllers are processed by microprocessors. Programmable common sense controllers also have the in-built PID controller commands. Because of the power and reliability of the PID controllers, these are historically used in technique manipulate programs.

\section{Working of PID Controller}

With use of low value easy $O N-O F F$ controller handiest manipulate states are possible, like absolutely $O N$ or absolutely $O F F$. It is used for limited manage application wherein these control states are sufficient for control objective. but oscillating nature of this manage limits its usage and therefore it's far being changed by using PID controllers.PID controller keeps the output such that there is zero mistakes among system variable and set point/ desired output by using closed loop operations. PID uses 3 simple control behaviors which can be explained below. 


\section{P- Controller}

P controller other name is proportional controller given output that is proportional to error of current $e(t)$.It compared set point with (actual) real value.

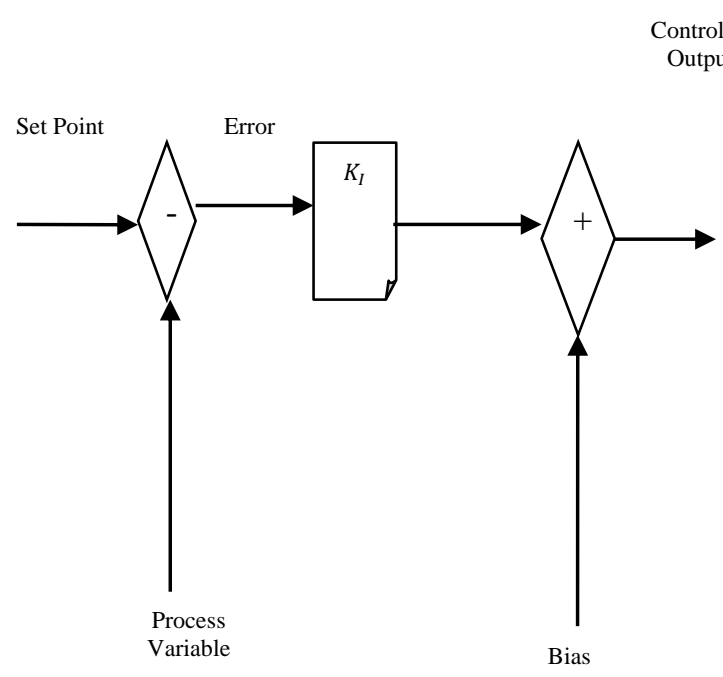

Fig.2 Basic design of P-controller

Output of controller get zero, when the value of error should be zero.

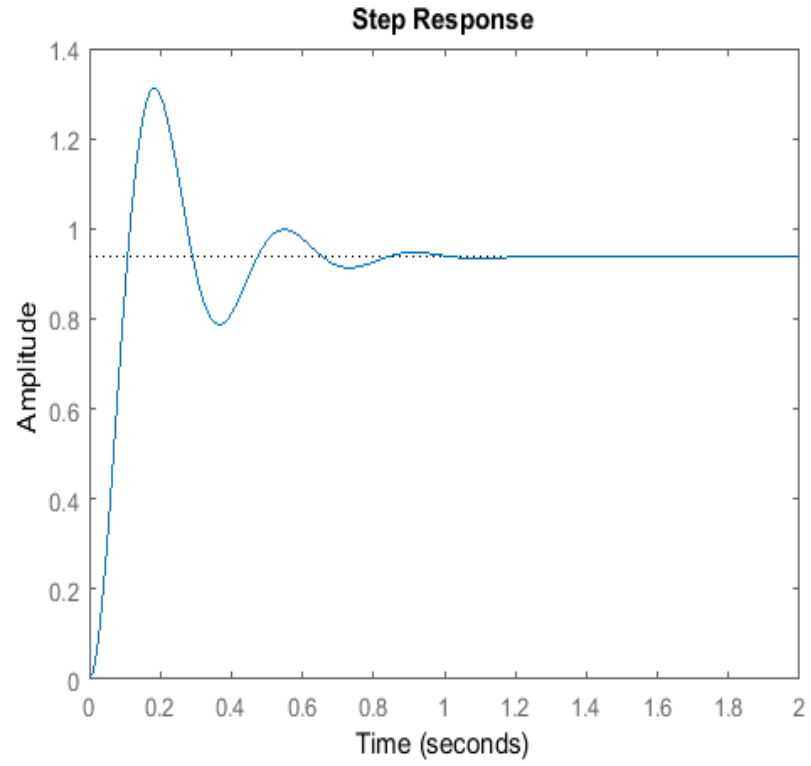

Fig.3 P-Controller Response
Appropriate to restraint of $p$-controller wherein there usually exists an offset among the method variable and set factor, I-controller is wanted, which gives important movement to do away with the steady country mistakes. It integrates the error over a time frame till blunders value reaches to zero. It holds the fee to final control tool at which error becomes zero. Integral manipulate decreases its output whilst bad mistakes takes place. It limits the rate of response and affects stability of the gadget. Velocity of the reaction is expanded by using reducing fundamental gain $K_{i}$.

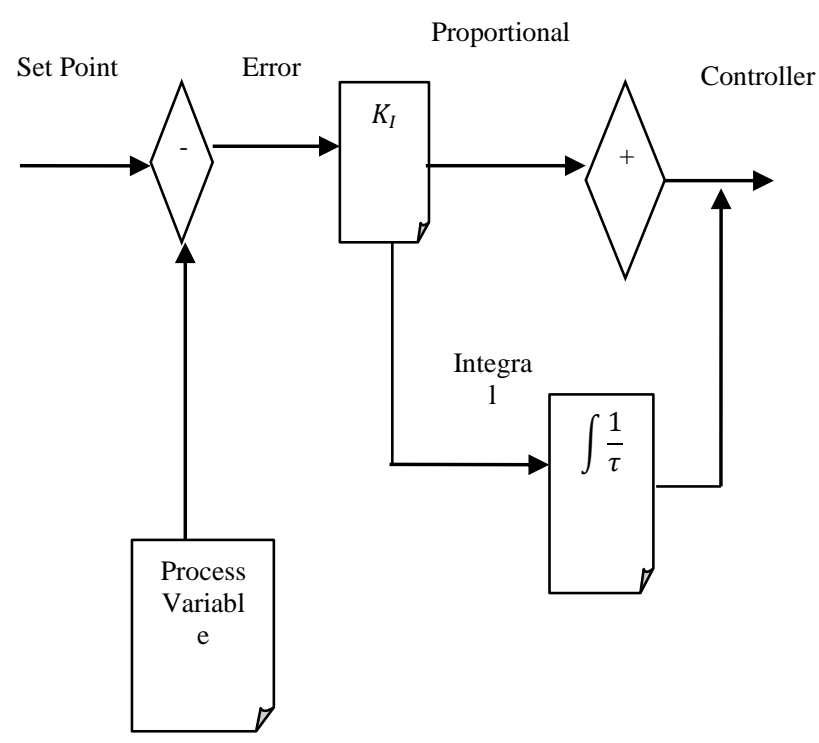

Fig. 4 Design of PI Controller

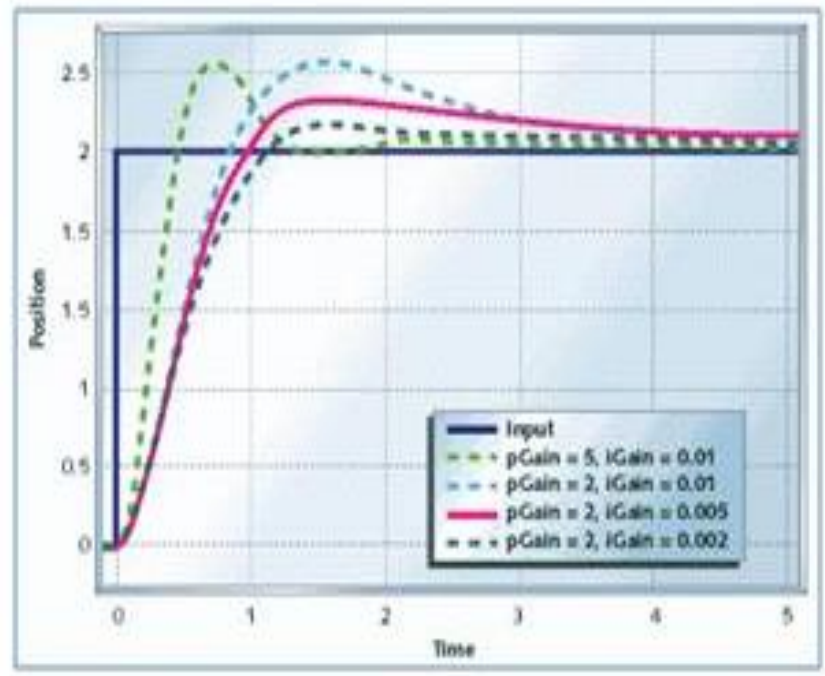

Fig.5 PI Controller Response

\section{I-Controller}


In above figure, because the benefit of the Icontroller decreases, steady nation blunders also goes on reducing. For most of the cases, PI controller is used in particular wherein excessive speed reaction is not required.

while the usage of the PI controller, I-controller output is restricted to somewhat variety to triumph over the essential wind up situations wherein necessary output is going on increasing even at 0 blunders state, due to nonlinearities within the plant.

\section{D-Controller}

I-controller doesn't have the capability to predict the future behavior of error. So it reacts normally once the set point is changed. $D$-controller overcomes this problem by anticipating future behavior of the error. Its output depends on rate of change of error with respect to time, multiplied by derivative constant. It gives the kick start for the output thereby increasing system response.

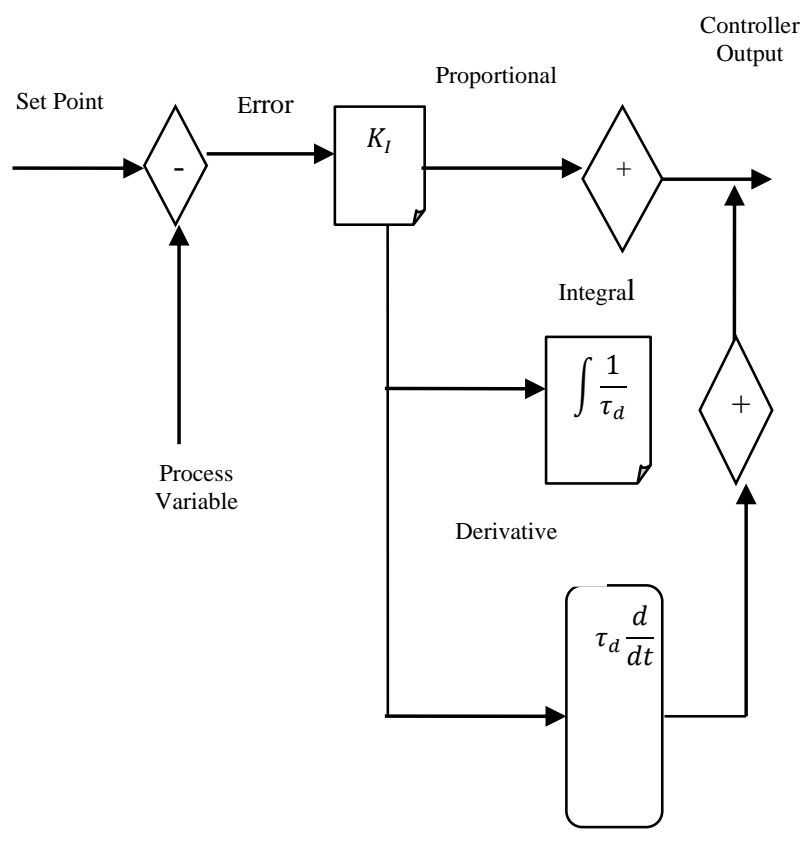

Fig. 6 Basic Representation of PID Controller

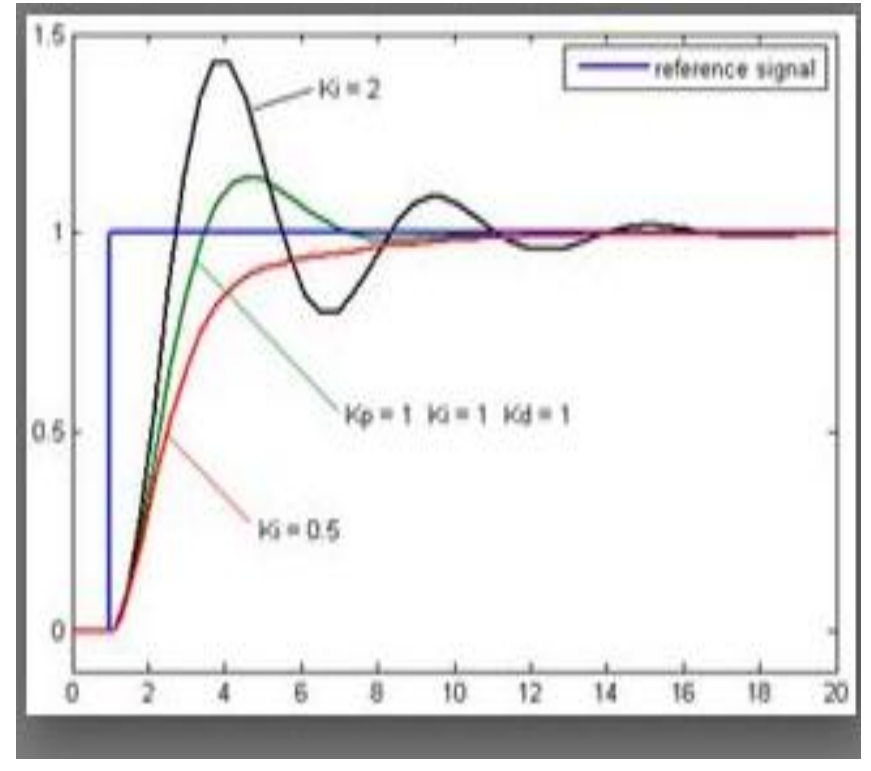

Fig.7 PID Controller Response

Within the above determine response of $D$ controller is greater, compared to PI controller and also settling time of output is decreased. It improves the stableness of gadget by means of compensating segment lag as a result of $I$ controller. Growing the by-product advantage will increase pace of reaction. So sooner or later we decided that by combining these three controllers we would get a favorable response for the system. Special manufacturers design special PID algorithms. The PID controller consists of three terms, namely proportional, required and derivative control. The combined operation of these 3 controllers offers a procedure control strategy. The PID controller manipulates the variables of the procedure, such as stress, speed, temperature, sliding, and many others. A number of programs use PID controllers in cascading networks where two or more PIDs are used to control.

\subsection{SMC-PID Control}

The target of tracking control is plain there a control law for obtain the appropriate input torque $\tau$ as the position vector $q$ shouldfollow the useful trajectory $q_{d}$.

$$
e=q_{d}-q
$$


In $S M C$, the sliding surface as define is equation (19) here the integral part of equation added to derivative term.

$$
s=\dot{e}+\lambda_{1} e+\lambda_{2} \int_{0}^{t} e d t
$$

Where $\lambda_{1}$ is diagonal positive definite matrix. So, $s=0$ defined as the stable sliding surface and $e \rightarrow 0$ as $t \rightarrow \infty$. We write the equation of robot dynamic equation in form of sliding surface (as filtered error) as given-

$$
M \dot{s}=-C s+f+\tau_{d}-\tau
$$

Where

$$
\begin{gathered}
f=M\left(\ddot{q}_{d}+\lambda_{1} \dot{e}+\lambda_{2} e\right)+C\left(\dot{q}+\lambda_{1} e\right. \\
\left.+\lambda_{2} \int_{0}^{t} e d t\right)+G
\end{gathered}
$$

Now, the control input cab be considered as follows

$\tau=\hat{f}+K_{v} s+K \operatorname{sgn}(s)$

Where

$\hat{f}=\widehat{M}\left(\ddot{q}_{d}+\lambda_{1} \dot{e}+\lambda_{2} e\right)+\hat{C}\left(\dot{q}+\lambda_{1} e+\right.$ $\left.\lambda_{2} \int_{0}^{t} e d t\right)+\hat{G}(19)$

Is estimation of $\mathrm{f}$ and $K_{v} s=K_{v} \dot{e}+K_{v} \lambda e+$ $K_{v} \lambda \int_{0}^{t} e d t$ in the outer $P I D$ tracking and $K_{v}, K$ are matrices of definite the diagonal and explained the stability of system conditions. There are $\operatorname{sign}(s)$ is defined as the sign function.

\section{2- DOF ROBOT MANIPULATOR}

The simulation example of 2 DOF Robot Manipulator is as follows:

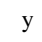

y

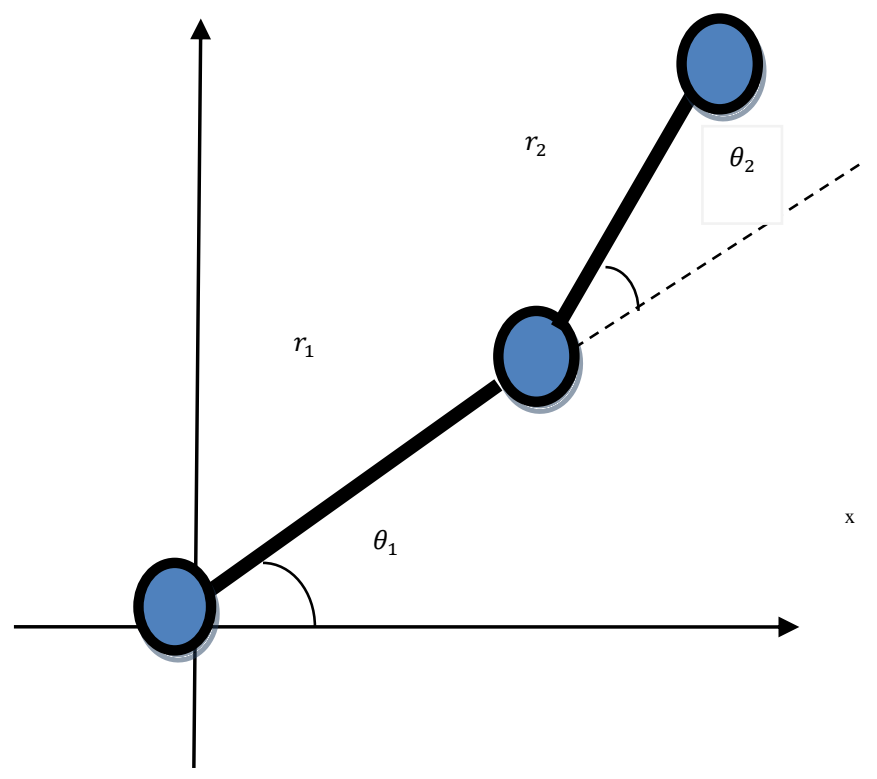

Fig.8 Simulation Example of 2 DOF Robot Manipulator

The Dynamic Equation of 2 DOF Robot Manipulator

$$
\begin{gathered}
{\left[\begin{array}{ll}
M_{11} & M_{12} \\
M_{12} & M_{22}
\end{array}\right]\left[\begin{array}{l}
\ddot{\theta}_{1} \\
\ddot{\theta}_{2}
\end{array}\right]\left[\begin{array}{cc}
-F_{12} \dot{\theta}_{2} & -F_{12}\left(\dot{\theta}_{1}+\dot{\theta}_{2}\right) \\
F_{12} \dot{\theta}_{1} & 0
\end{array}\right]\left[\begin{array}{c}
\dot{\theta}_{1} \\
\dot{\theta}_{2}
\end{array}\right]} \\
+\left[\begin{array}{l}
G_{1} g \\
G_{2} g
\end{array}\right]=\left[\begin{array}{l}
u_{1} \\
u_{2}
\end{array}\right]
\end{gathered}
$$

$$
\begin{gathered}
M_{11}=\left(m_{1}+m_{2}\right)^{*} r_{1}{ }^{\wedge} 2+m 2^{*} r 2^{\wedge} 2 \\
+2^{*} \mathrm{~m} 2^{*} \mathrm{r} 1^{*} \mathrm{r} 2^{*} \cos \left(\theta_{2}\right) \\
M_{12}=m 2^{*} r 2^{\wedge} 2+m 2^{*} r 1^{*} r 2^{*} \cos (\theta 2)
\end{gathered}
$$

$M 22=m 2^{*} r 2^{\wedge} 2$;

$$
\begin{gathered}
F 12=m 2^{*} r 1^{*} r 2^{*} \sin (\theta 2) ; \\
g 1=(m 1+m 2)^{*} r 1^{*} \cos (\text { theta } 2) \\
+m 2^{*} r 2^{*} \cos (\theta 1+\theta 2) ; \\
g 2=m 2^{*} r 2^{*} \cos (\theta 1+\theta 2)
\end{gathered}
$$




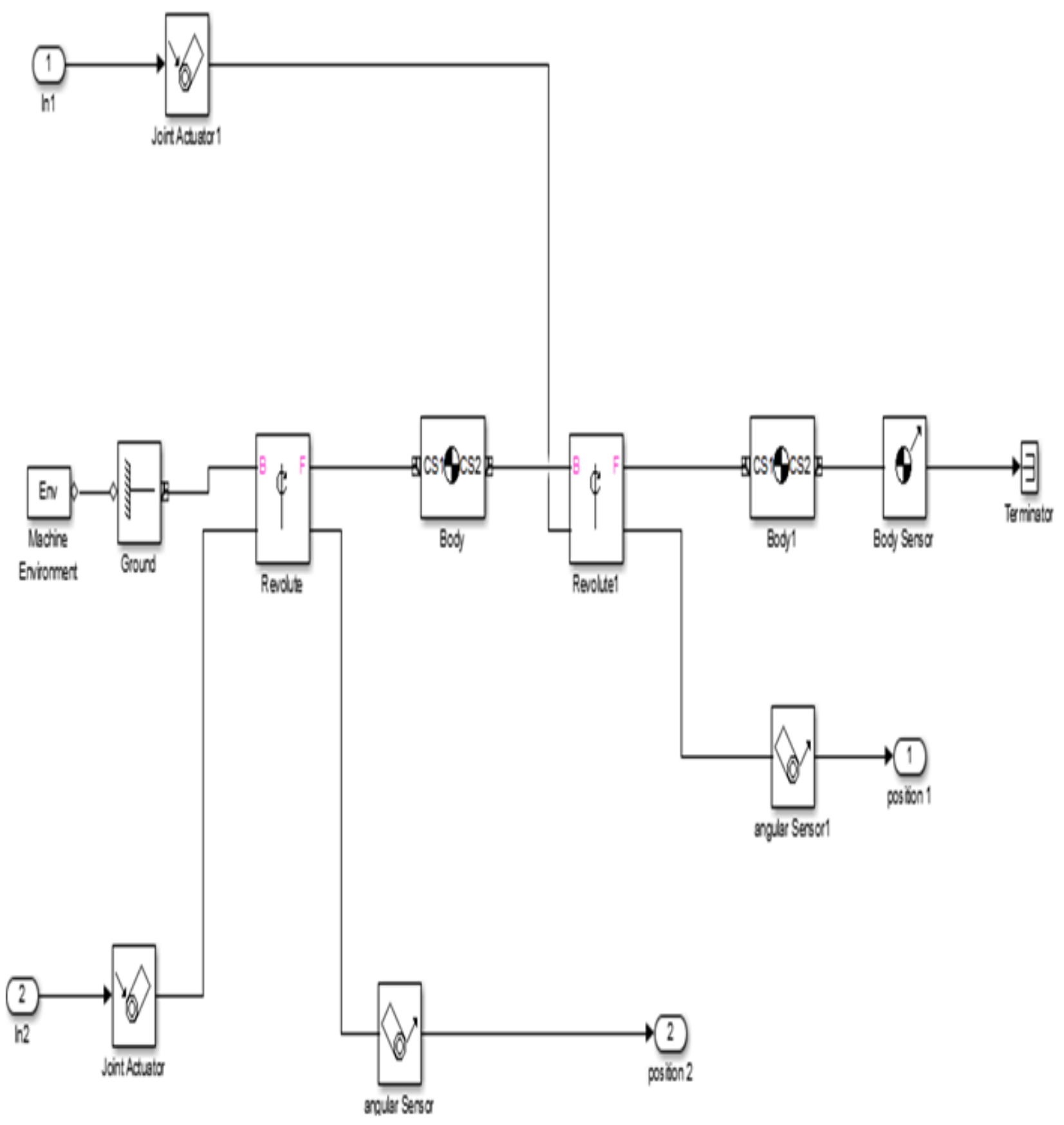

Fig. 9 Simulink model of 2 DOF robot 


\section{RESULTS AND PRESENT COMPARATIVE PERFORMNCE ANAYSIS}

The both PID and SMC controller are employs on 2 DOF robots Manipulator. The recital of $S M C$ controller is finer toward PID. SMC controller outputs are more accurate as compare to PID.

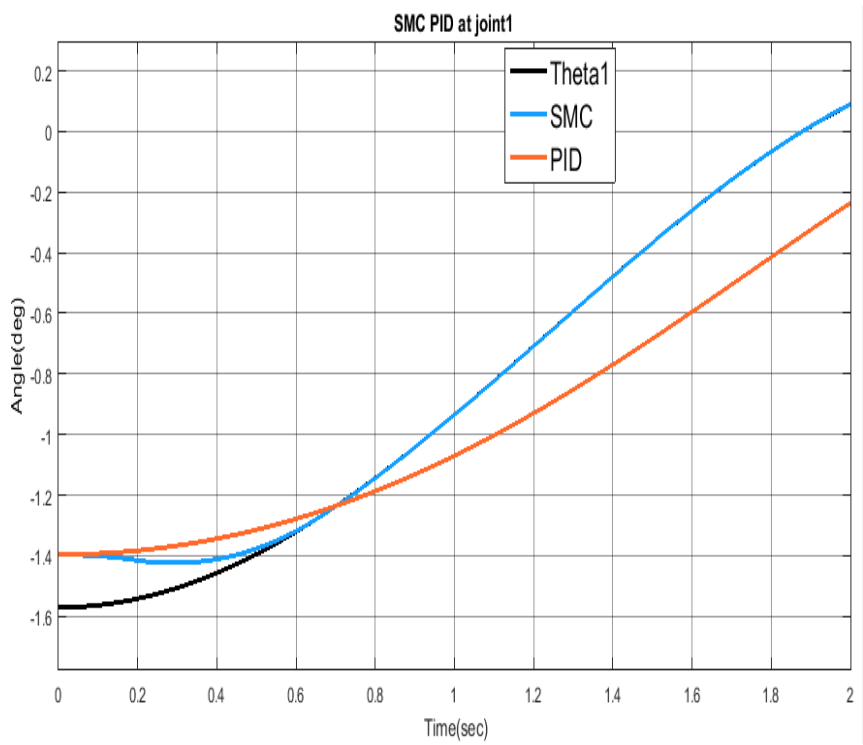

Fig. 10 Comparative results of SMC and PID at joint1

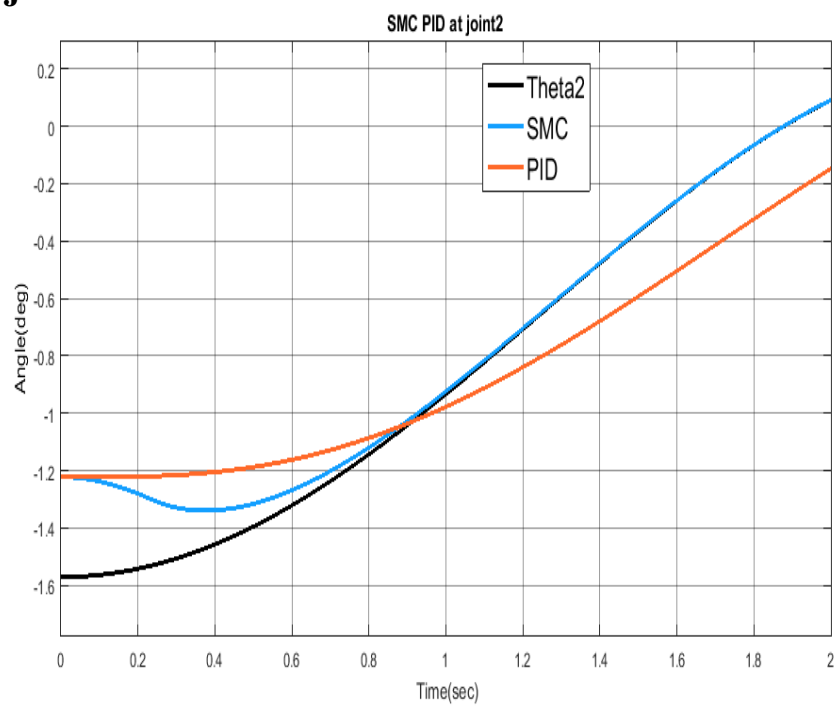

Fig. 11 Comparative results of SMC and PID at joint1

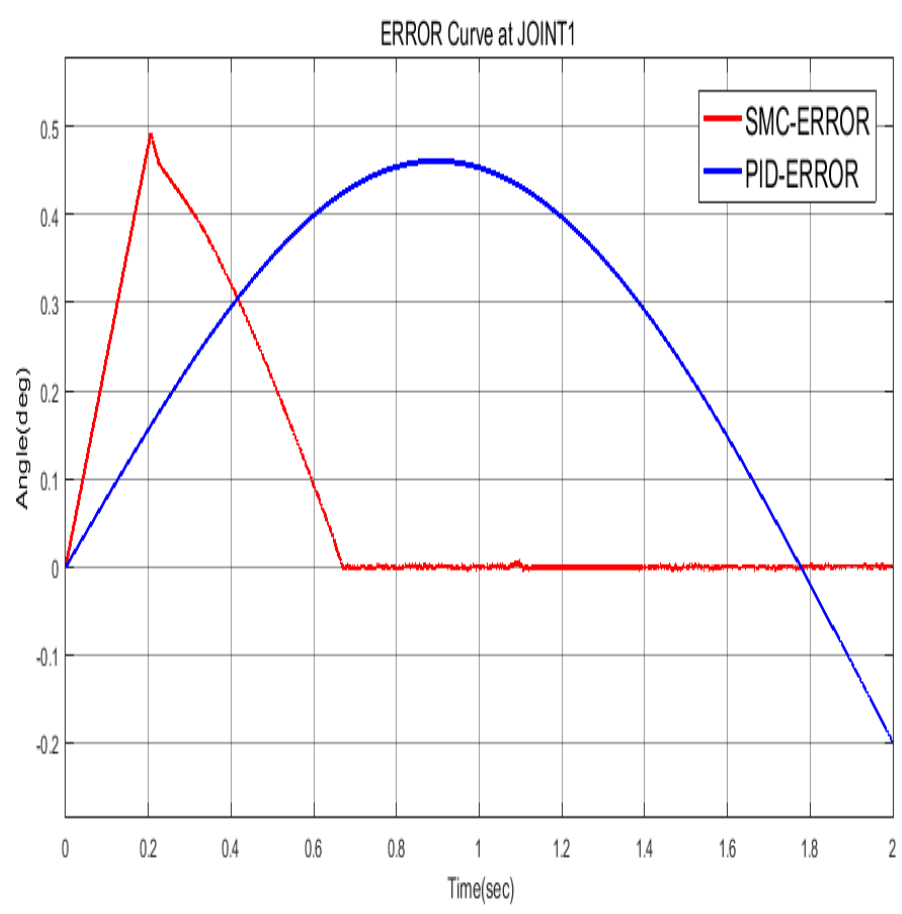

Fig.12 Error curve

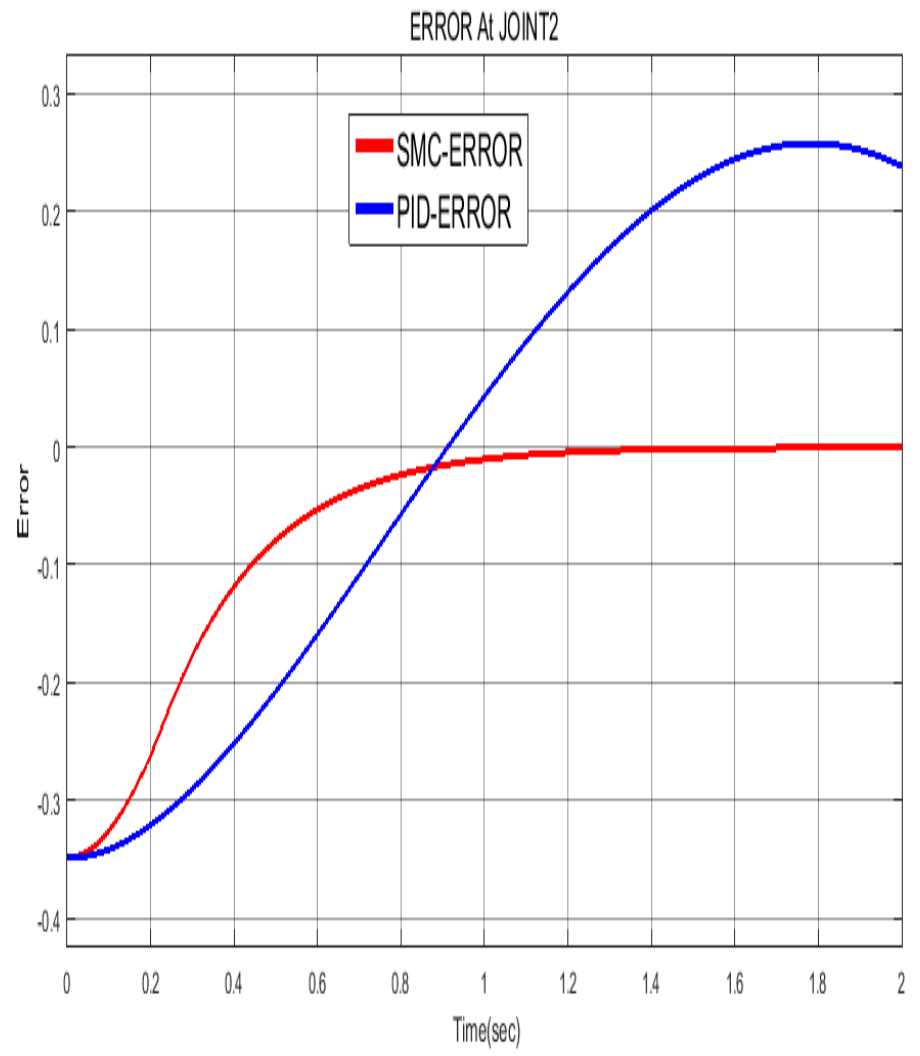

Fig.13 Error curve 


\section{Www.rspsciencehub.com}

The figure 12 and 13 shows error curve of both controllers. The error is in $S M C$ is very less since compare to PID controller.

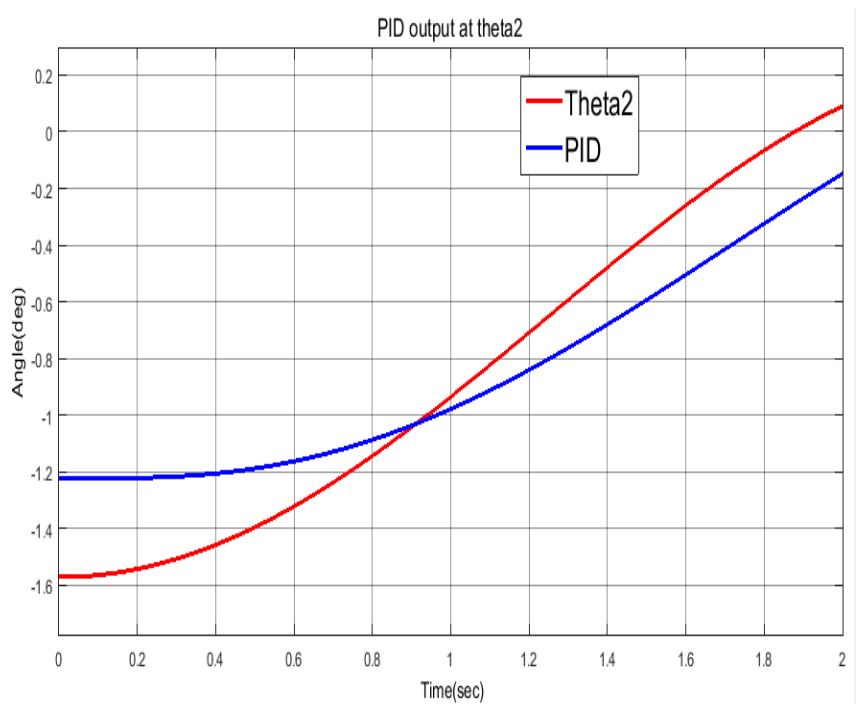

Fig. 14 Result of PID at joint

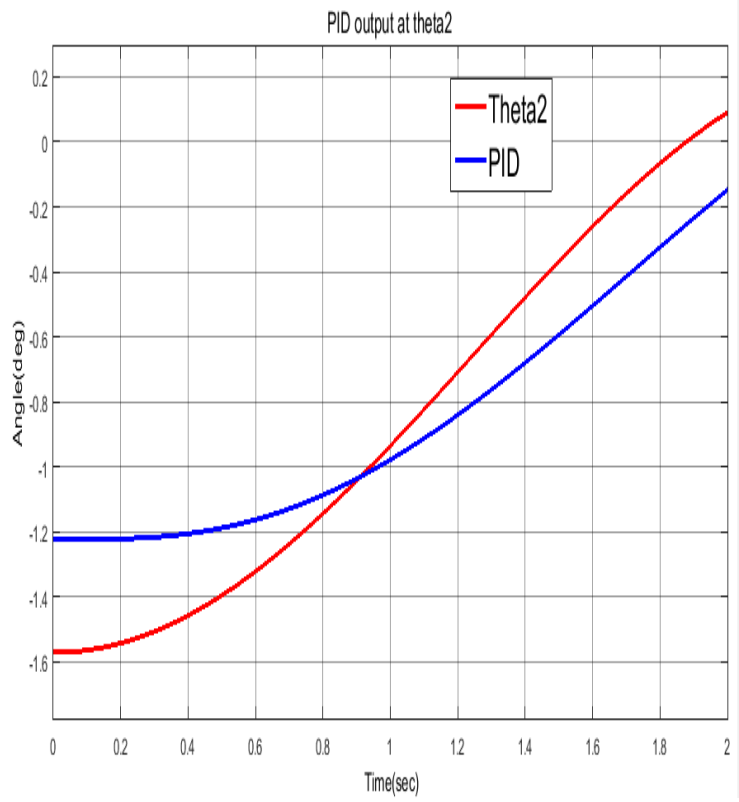

Fig. 15 Result of PID at joint

The results shows that figure 14 and 15 the angle at joint 1 and joint 2 follow the path of reference angle theta1 and theta2.
Volume 02 Issue 06 June 2020

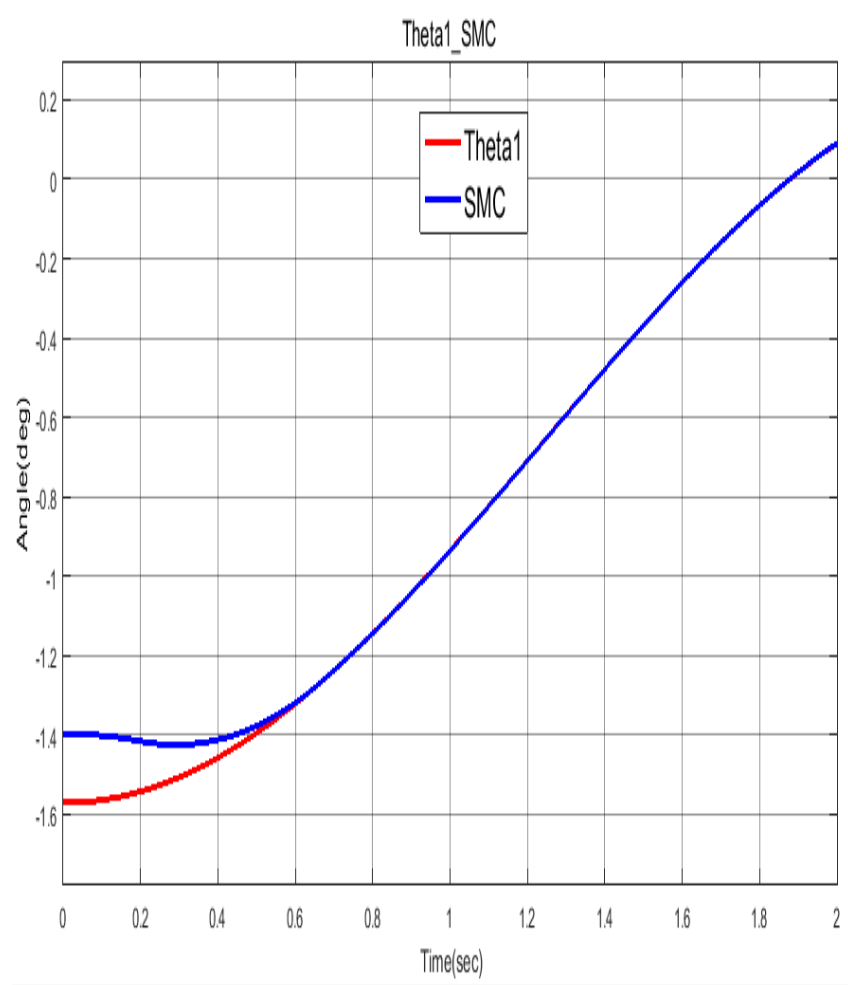

Fig.16 Result of SMC at joint 1

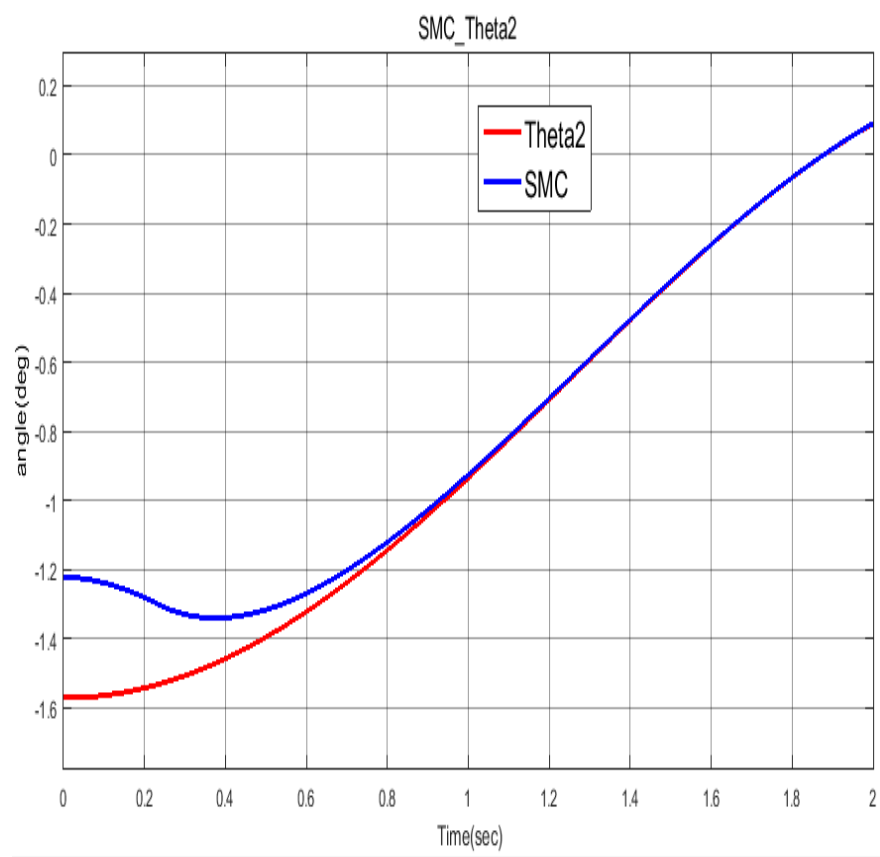

Fig.17 Result of SMC at joint 2

From figure 16 and 17 it is clear to facilitate output of $S M C$ controller track reference signal in very accurately and in well manner. 


\section{Conclusion \& Future Aspects:}

A two links robotic manipulator was studied and its dynamics was modeled using Lagrange mechanics. A robust control based on the conventional $S M C$ is introduced to control the motion of the robot at specific position for pick and place activities along with PID control scheme. The obtained results have shown that the SMC outperform the PID controller in term of fast and robust response though with higher control signal. The high joint speeds in case of $S M C$, which are a consequence of high control signal in this case, are required for rapid movement of the links. In this work $S M C$ is analyzed to be of better performance than $P I D$. It is expected that adaptive $S M C$ control may lead to a better response which is left for future work.

\section{References:}

[1] M. Young, The .Technical Writer's Handbook, Mill Valley, CA: University Science, 1989.

[2] A. Okubanjo, O. Oyetola, M.Osifeko, O. Olaluwoye and P. Alao modeling of 2-DOF robot arm and control.

[3] David, I., and G. Robles. "PID control dynamics of a Robotic arm manipulator with two degrees of Freedom," Control de Process y Robotic (2012): 1-7...

[4] M. Baccouch and S. Dodds, A two-link manipulator: simulation and control design, University of Nebraska at Omaha, 2012.

[5] C.T. Lin, K.C. Liu, C.W. Chung, and Y. Chang, Design of Sliding Mode Controller for Two-Link Robot Manipulator, in Robot, Vision and Signal Processing (RVSP), 2011 First International Conference on, 2011, pp. 9093.

[6] F. Piltan, S. Emamzadeh, Z. Hivand, F. Shahriyari, and M. Mirazaei, PUMA-560 Robot Manipulator Position Sliding Mode Control Methods Using MATLAB/SIMULINK and Their Integration into Graduate/Undergraduate Nonlinear Control, Robotics and MATLAB Courses, International Journal of Robotics \& Automation, vol. 3, no. 3, pp. 106150, 2012.

[7] F. Piltan and N. B. Sulaiman, Review of sliding mode control of robotic manipulator, World
Applied Sciences Journal, vol. 18, no. 12, pp. 1855-1869, 2012.

[8] PALM R, DRIANKOV R, HELLENDOORN H. Model Based, and Fuzzy Control: Fuzzy Gain Schedulers and sliding mode, Fuzzy Controllers Springer-Verlag Berlin Heidelberg, 1997.

[9] LO J C, KUO Y H. Decoupled Fuzzy Sliding Mode Control, IEEE Trans on Fuzzy systems, 1998, 6(3): 426-435...

[10] WANG L K, LEUNG H F, TAM K S. A Fuzzy Sliding, .Controllers for Nonlinear Systems. IEEE Trans. Ind. Elec., 2001, 48(1): 32-37...

[11] HA Q P, NGUYEN Q H, RYE D C, DURRANT-WHYTE H F. Fuzzy Sliding Mode Controllers with Applications. IEEE, Trans. Ind. Elec., 2001, 48(1).

[12] LIN Z, ZHU Q, YAN Y. Variable Structure Control Based on Fuzzy Law for under actuated Robot Manipulators. IEEE Int.,. Conf. on Mechatronics and Automation, 2006: 1908-1913.

[13] LIN C M, MON Y J. Decoupling Control by Hierarchical Fuzzy Sliding Mode Controller. IEEE Trans. Cont. Sys. Technology, 2005, 13(4): 593598.

[14] HUANG Y C, LI T H S. Fuzzy Terminal Sliding Mode Controller for Robotic Manipulators. IEEE Int. Conf. on Mechatronics, 2005: 858-863.

[15] HA Q P, RYE D C, DURRANT-WHYTE H F. Fuzzy Moving Sliding Mode Control with Application to Robotic Manipulators, Automatic a, 1999, 35: 607-616.

[16] JAVAHERI H, VOSSOUGHI G R. Sliding Mode Control with Online Fuzzy Tuning: Application to a Robot Manipulator., IEEE Int. Conf. on Mechatronics \& Automation, 2005: $1357-1362 \ldots$

[17] KUO T C, HUANG Y J. a Sliding mode and PID Controller Design for Robot Manipulator. IEEE Conf. On Computational Intelligence in Robotics and Automation, 2005: 625-629.

[18] EKER I. Sliding mode. Control with PID sliding surface and experimental application to an electromechanical plant. ISA Trans, 2006, 45,(1): 109-118.

[19].PERRUQUETTI W, BARBOT J P. Sliding Mode Control in Engineering. Marcel Dekker, Inc. New York 2002.

[20].Hsu, F.Y. and L.C. Fu., 1995. Nonlinear control of robot manipulators using adaptive fuzzy 
sliding mode control. IEEE Conference on Intelligent Robots and Systems, pp: 156-161.

[21] Yao-Chu Hsueh, Shun-Feng Su and Wen-June Wang. 2008. Self tuning sliding mode controller design for class a of nonlinear control systems. International Conference on Systems Man and Cybernetics, pp: 2337-2342.

[22]Shrey Kasera, Amit Kumar and L.B Prasad,"Trajectory Tracking of 3 DOF Industrial Robot Manipulator by Sliding Mode Control"“, Proc. of $20174^{\text {th }}$ IEEE Uttar Pradesh Section International Conference on Electrical ,Electronics \& Computer Engineering (UPCON-2017)pp.364369,26-28 OCT,2017, GLA Mathura.

[23] Shrey Kasera, Amit Kumar and L.B Prasad,"Anaysis of Chattering Free Improved Sliding Mode Control", Proc. of 2017.

[24] International Conference on Innovations. Information Embedded \& Communication System (ICIIECS), Vol.3, pp. 936-941, March 17-18, 2017, Coimbatore, India...

[25]Mohammed, Amin \& Eltayeb, .Ahmed. (2018),. Dynamics and Control of a Two-link Manipulator using PID and Sliding Mode Control. 1-5.10.1109/ ICCCEEE, 2018.8515795.

[26] A. C. Emmanuel and H. C. Inyiama, "A survey of controllers design methods for a robot manipulator in harsh environments," vol. 3, no. 3, pp. 64-73, 2015.

[27] Kabir Usman, et al."Performance Analysis of PID, PD and Fuzzy Controllers for Position Control of 3 DOF Robot Manipulator" ArXiv preprint arXiv: 1910.12076 (2019). 